\title{
The Simulation of Annular Dark Field Images of InAs/InP Quantum Dots
}

\author{
M. D. Robertson, * J. C. Bennett,* M. M. J. Burns, ${ }^{*}$ and D. Currie**
}

* Department of Physics, Acadia University, Wolfville, Nova Scotia, B4P 2R6, Canada ** Acadia Centre for Mathematical Modelling and Computation, Acadia University, Wolfville, Nova Scotia, B4P 2R6, Canada

Annular dark field (ADF) images of complete InAs quantum dots (QDs) in an InP matrix have been simulated in order to study the effects of strain and composition on image contrast. The QDs had a base radius of $2.5 \mathrm{~nm}$, a height of $3.0 \mathrm{~nm}$ and were situated on a $0.5 \mathrm{~nm}$ InAs wetting layer. The elastic displacement fields, arising from the 3.1\% lattice mismatch between InAs and InP, were simulated using finite-element methods and the appropriate anisotropic elastic constants were used for each material [1]. Figures 1A and 1B are the horizontal (x) and vertical (z) displacements of the $\mathrm{QD}$ and surrounding material for a plane through the centre of the QD. The magnitude of the displacements is greatest at the edge of the dot and a maximum value of $0.05 \mathrm{~nm}$ is observed.

A $10 \mathrm{~nm} \times 10 \mathrm{~nm} \times 10 \mathrm{~nm}$ unit cell, comprised of 39168 atoms, was constructed in the [110] zone axis projection where the atoms were displaced from their equilibrium positions in three dimensions in accordance with the finite-element calculations. The ADF images were simulated using the program AUTOSLIC written by Kirkland [2] modified to include a calculation of the incident electron probe as well as an improved sorting algorithm. The effects of thermal vibrations were included using the frozen phonon approximation [2]. The simulations were performed on a parallel-computing cluster. Each image displayed in Fig. 2 is an average of four separate runs and represents about 240 processor-days of computation time (1.4 GHz, AMD64 Opteron 250 processors). The microscope parameters were typical of a JEOL $2100 \mathrm{~F} \mathrm{TEM}$ and the values used in the calculations were: $\mathrm{E}=$ $200 \mathrm{kV}, \mathrm{C}_{\mathrm{s}}=0.5 \mathrm{~mm}$, defocus $=87 \mathrm{~nm}$ and a $6 \mathrm{mrad}$ diameter aperture yielding a focused electron probe with a FWHM of $0.25 \mathrm{~nm}$. Each point in the images was evaluated using 2048 x 2048 arrays for the wavefunction and FFT, and the inner and outer detector angles were $77 \mathrm{mrad}$ and $170 \mathrm{mrad}$, respectively. Figure 2A was calculated at the exact [110] zone axis condition and it is observed that image intensity has been reduced at the edges of the QD where the atomic displacements due to strain are greatest. Hence, strain interferes with the simple interpretation of compositional dependent contrast in HAADF images of QDs. Figure 2B is for the same QD geometry except that it has been rotated by 4 degrees about a horizontal line running through the centre of the QD. In this case, strain contrast has been suppressed, particularly in the wetting layer underneath the QD. Figures 3A and 3B are line scans of the image intensity through the centre of the QD where the simulations included the effects of composition only, composition and strain, and strain only. The effects of strain are seen to be reduced when the sample is tilted off of the exact zone axis condition.

In conclusion, the simulations indicate that that the effects of strain on image contrast must be considered when attempting to extract quantitative compositional information from on-axis ADF images of InAs QDs embedded in an InP matrix. These effects can be minimized in off-axis orientations.

[1] M. D. Robertson et al., Ultramicroscopy 103 (2005).

[2] E. J. Kirkland, Advanced Computing in Electron Microscopy, Plenum Press, New York, (1998).

[3] This work was supported by the Canada Research Chairs program and NSERC. 

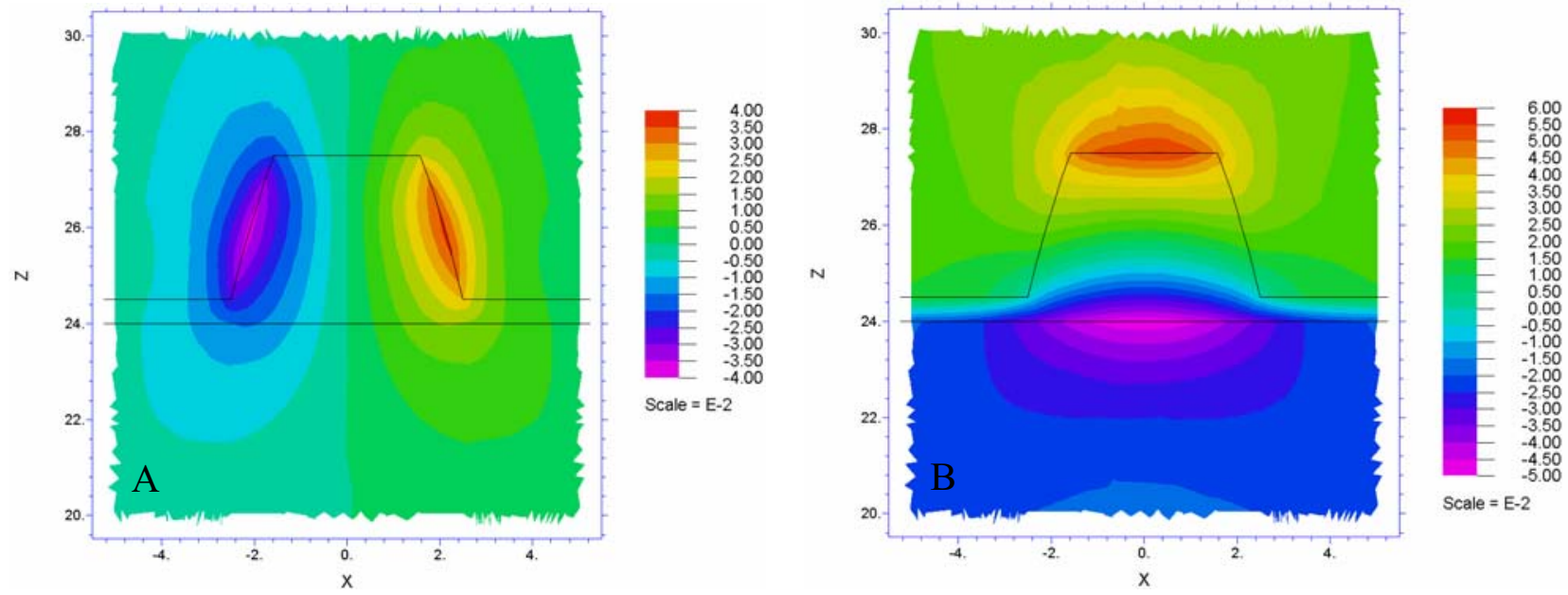

Fig. 1. Finite element simulations of the displacement field of the QD. A. The X-component of the displacement. B. The z-component of the displacement. All units are in nanometers.
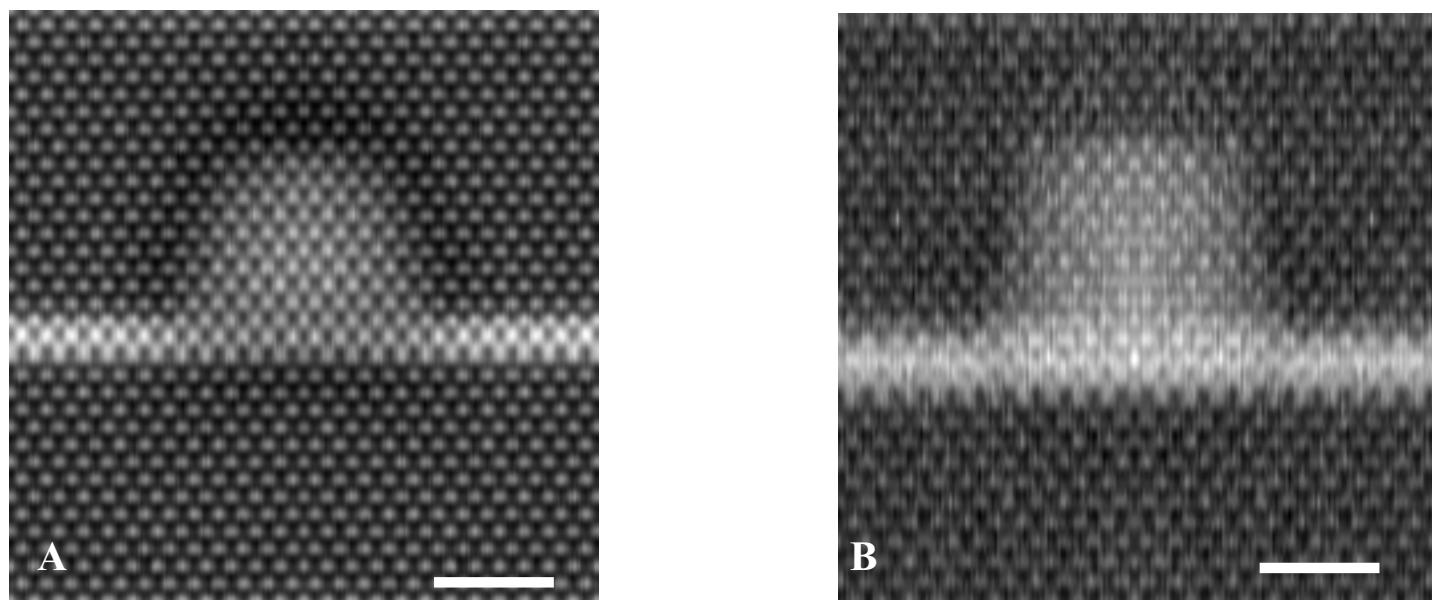

Fig. 2. HAADF simulations of the QD for detector angles ranging from 77 to $170 \mathrm{mrad}$. A. Specimen orientated at exactly the [110] zone axis. B. Specimen rotated by 4 degrees about a horizontal line through the centre of the QD. Scale bar $=2 \mathrm{~nm}$.
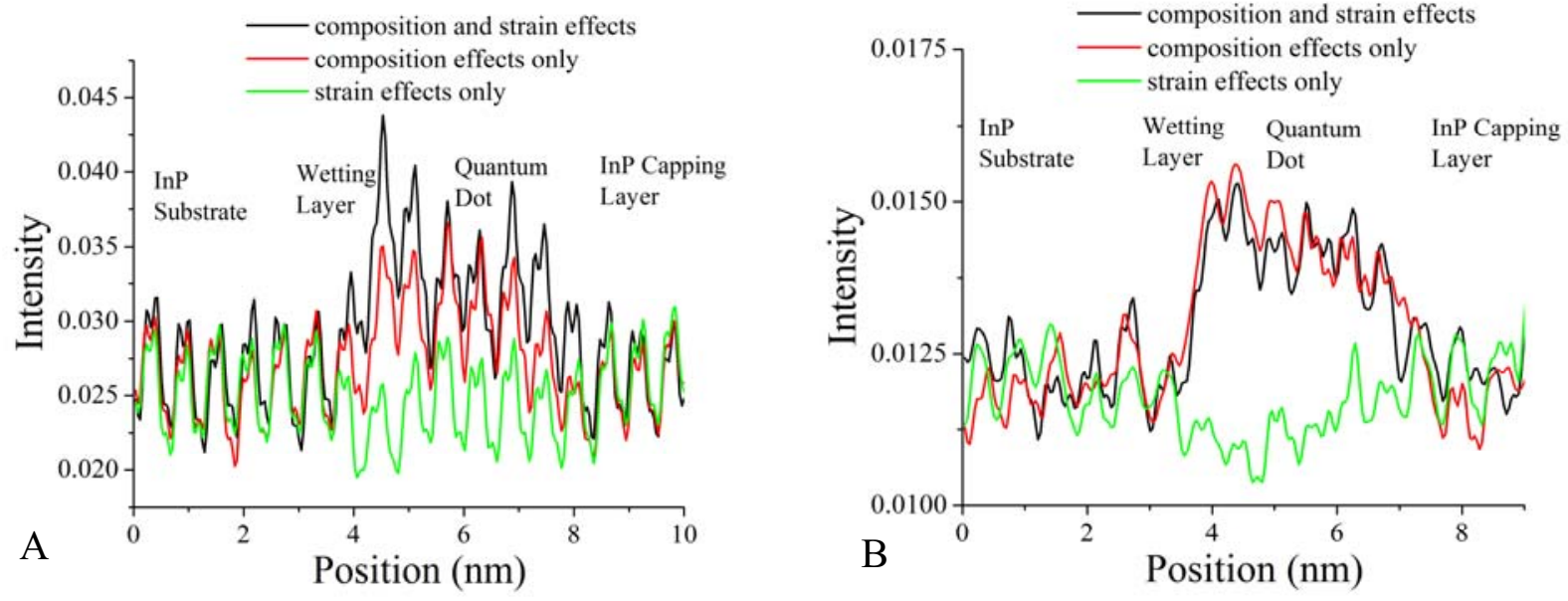

Fig. 3. Line scans of the intensity taken vertically through the centre of the QD. A. Specimen orientated at exactly the $[110]$ zone axis. B. Specimen rotated by 4 degrees as in Fig. 2 B. 